\title{
Design, Simulation and Evaluation of Kinematic Alternatives for Insertable Robotic Effectors Platforms in Single Port Access Surgery
}

\author{
Jienan Ding, Kai Xu, Roger Goldman, Peter Allen, Dennis Fowler, Nabil Simaan
}

\begin{abstract}
This paper presents the task specifications for designing a novel Insertable Robotic Effectors Platform (IREP) with integrated stereo vision and surgical intervention tools for Single Port Access Surgery (SPAS). This design provides a compact deployable mechanical architecture that may be inserted through a single $\varnothing 15 \mathrm{~mm}$ access port. Dexterous surgical intervention and stereo vision are achieved via the use of two snake-like continuum robots and two controllable CCD cameras. Simulations and dexterity evaluation of our proposed design are compared to several design alternatives with different kinematic arrangements. Results of these simulations show that dexterity is improved by using an independent revolute joint at the tip of a continuum robot instead of achieving distal rotation by transmission of rotation about the backbone of the continuum robot. Further, it is shown that designs with two robotic continuum robots as surgical arms have diminished dexterity if the bases of these arms are close to each other. This result justifies our design and points to ways of improving the performance of existing designs that use continuum robots as surgical arms.
\end{abstract}

\section{INTRODUCTION}

Robotic assistance in Minimally Invasive Surgery (MIS) extended the capabilities of surgeons via improved precision, dexterity, and computer assistance[1, 2]. Recently, novel SPAS and Natural Orifice Trans-luminal Endoscopic Surgery (NOTES) have been investigated [3-6] for their potential benefits in reducing patient trauma and shortening their recovery time compared to MIS. However, SPAS and NOTES also set strict requirements for instrument miniaturization, dexterity, and collision avoidance between surgical tools operating in confined spaces. Existing surgical robots for MIS cannot satisfy these requirements due to either dexterity deficiency or the size of their actuation mechanisms that prohibit a multitude of arms from operating through a single port. Therefore, to date, SPAS is still limited to a small number of academic centers [7-9].

Manuscript received September 15, 2009. This work was supported by grant No. 5R21EB007779-02 from the National Institute of Health (NIH). Nabil Simaan was also funded through NSF Career award \#IIS-0844969.

Jienan Ding, Kai Xu and Nabil Simaan (“corresponding author) are with the Department of Mechanical Engineering, Columbia University, New York, NY 10027, USA. \{dj2253, kx2102,“ns2236\}@columbia.edu. In September 2009, Kai Xu joined UM-SJTU Joint Institute, Shanghai Jiao Tong University (k.xu@ @jtu.edu.cn).

Roger E. Goldman (reg2117@columbia.edu) is with Department of Biomedical Engineering and College of Physicians \& Surgeons, Columbia University, New York, NY 10027, USA.

Peter K. Allen (allen@ cs.columbia.edu) is with Department of Computer Science, Columbia University, New York, NY 10027, USA

Dennis L. Fowler (dlf91@ columbia.edu) is with Department of Surgery, Columbia University New York, NY 10032, USA.
Surgeons and engineers tried to overcome the single port constraint by using multi-port trocars (Tripot from Advanced Surgical Concepts, Wicklow, Ireland) and single incision laparoscopic surgery port from Covidien, Inc., which allow multiple instruments to pass through a single port. Others (Realhand from Novare and Cambridge Endo) used instruments which can bend to avoid the collision between the operator hands [10]. Animal studies of single port access laparoscopic cholecystectomy have been carried out using these instruments [11]. However this approach requires surgeons to operate with crossed hands, while relying on exceptional hand-eye coordination and substantial training.

Other researchers developed robotic assistance tools for NOTES. Abbott, [4], developed a wire-actuated dual-arm robotic system for NOTES which has $16 \mathrm{DoF}$ and a diameter larger than $20 \mathrm{~mm}$. Phee et. al., [6], presented a 9 DOF $\varnothing 22 \mathrm{~mm}$ dual-arm robot. Lehman et. al. , [5], developed NOTES robot that may be inserted into the abdomen via a $\varnothing 20 \mathrm{~mm}$ overtube. This robot requires surgeon intervention to switch it from a folded configuration to a working configuration. It is also fixed to the abdomen using external magnets. More recently, [12] introduced a novel concept of reconfigurable self-assembling robot for NOTES. This concept yet has to be experimentally proven.

There is a need for self-deploying robots that provides adequate dexterity in a size smaller than $20 \mathrm{~mm}$ diameter, while supporting 3D vision feedback during all operation phases (deployment and work). The aim of our ongoing investigation is to design new robotic platforms that are able to meet the challenges of SPAS and subsequently NOTES while satisfying these needs.

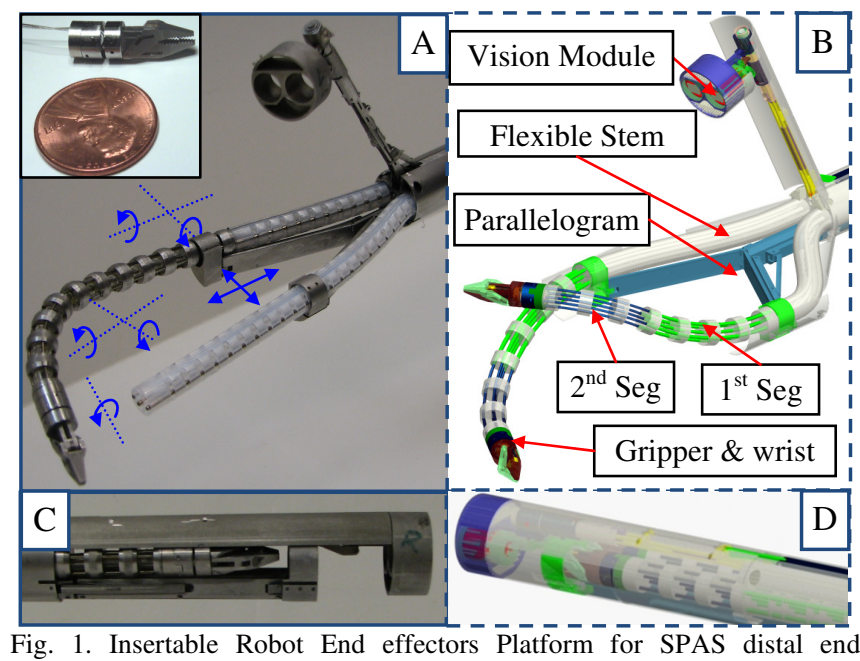
architecture and prototype. 
The contribution of this paper is in presenting a unique IREP for SPAS, which has 21 actuated joints with a controllable 3D stereo vision module and two dexterous arms having 8 joints. The unique feature of the IREP is that it can be folded into $\varnothing 15 \mathrm{~mm}$ configuration as shown in Fig. 1 . Design objectives are presented with a comprehensive model of the kinematics and statics of the IREP. The analysis presented here compares the dexterity of the IREP to other design alternatives. A justification for the current design is derived from simulations of a sample suturing task. The ability to triangulate two arms to the same position is also compared between the IREP and other designs.

\section{DESIGN SPECIFICATION}

The IREP was first presented in[13]. The workspace of the IREP has been validated through simulation and it was shown that the proposed design in Fig. 1 is capable of covering a

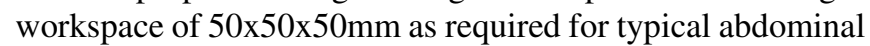
procedures such as cholystectomy. Results of [14, 15] provided the required force and torques for typical abdominal procedure (Table 1). Other design specifications such as maximal translation velocity and precision were obtained from our surgical team members (Table 1 Distal snake robot). The design specifications of the actuation unit are calculated from IREP kinematics and static model and will be discussed in following section. In addition, the system should also be designed with quick-connect interfaces that allow fast instrument exchange during surgery.

TABLE 1 DESIGN SPECIFICATION OF IREP

\begin{tabular}{|c|c|c|c|}
\hline \multicolumn{4}{|c|}{ Distal Snake Robot } \\
\hline $\begin{array}{c}\text { Workspace } \\
(\mathrm{mm})\end{array}$ & Working Speed & Working load & Precision \\
\hline $\begin{array}{c}\mathrm{X}: \pm 25, \mathrm{Y}: \pm 25 \\
\mathrm{Z}: \pm 30\end{array}$ & $\begin{array}{c}30 \mathrm{~mm} / \mathrm{s} \\
60^{\circ} / \mathrm{s}\end{array}$ & $\begin{array}{c}\text { Force: } 2 \mathrm{~N} \\
\text { Moment: } 60 \mathrm{mNm}\end{array}$ & $\begin{array}{l} \pm 0.25 \mathrm{~mm} \\
\pm 1^{\circ}\end{array}$ \\
\hline \multicolumn{4}{|c|}{ Base Module of Actuation Unit } \\
\hline & Travel & Speed & Load \\
\hline Gross trans & $\pm 50 \mathrm{~m}$ & $60 \mathrm{~mm} / \mathrm{s}$ & $300 \mathrm{~N}$ \\
\hline 5Bar Mech & $\pm 5 \mathrm{~mm}$ & $5 \mathrm{~mm} / \mathrm{s}$ & $100 \mathrm{~N}$ \\
\hline Camera & $\pm 5 \mathrm{~mm}$ & $5 \mathrm{~mm} / \mathrm{s}$ & $20 \mathrm{~N}$ \\
\hline \multicolumn{4}{|c|}{ Snake Module of Actuation Unit } \\
\hline & Travel & Speed & Load \\
\hline Snake & $\pm 8 \mathrm{~mm}$ & $30 \mathrm{~mm} / \mathrm{s}$ & $60 \mathrm{~N}$ \\
\hline Wrist & $\pm 5 \mathrm{~mm}$ & $5 \mathrm{~mm} / \mathrm{s}$ & $30 \mathrm{~N}$ \\
\hline Gripper & $\pm 5 \mathrm{~mm}$ & $5 \mathrm{~mm} / \mathrm{s}$ & $20 \mathrm{~N}$ \\
\hline
\end{tabular}

\section{THE IREP DESIGN}

The IREP has 21 actuators that drive its two dexterous arms, vision module, and two five-bar (parallelogram) mechanisms that allow self deployment of the dexterous arms and adjustment of the distance between the bases of these two arms. During insertion, the IREP folds into a cylindrical configuration with a diameter of $\varnothing 15 \mathrm{~mm}$ Fig. 1. Insertion into the patient abdomen is carried out using a trocar at the umbilicus. After insertion, the IREP deploys two dexterous snake-like arms equipped with distal wrists and grippers. A third arm is also deployed with a 3D vision module.

Each dexterous arm has 7DoF, consisting of a 4 DoF two-segment continuum snake-like robot, a 1 DoF wrist, a gripper and a two DoF five-bar parallelogram mechanism that deploys each continuum robot. The diameter of individual continuum snake-like robot is $6.4 \mathrm{~mm}$. The choice of continuum flexible robots using NiTi backbones was motivated by the inherent safety of flexible robots in manipulating organs and their enhanced down-scalability.

This design is a modification of the four-backbone design in[16]. Each continuum robot arm has a single primary backbone and four equidistant and circumferentially distributed secondary backbones. The advantage of this design is in the simplicity of actuation since each backbone can be pulled while the other radially-opposing backbone can be pushed by the same amount. This modification eliminates the need for software kinematic coupling between opposing backbones - a feature that simplifies deployment and homing of these robots.

The wrist is a wire-driven joint that allows independent rotation of the gripper about its longitudinal axis. The independent axis adds critical dexterity to suturing tasks in confined spaces. While it is possible to provide rotation about the axis of the gripper by using the continuum robots as constant velocity joints through careful coordination of actuation of all backbones [16], the use of an independent wrist simplifies the control and improves dexterity (see Section V).

The vision module includes two CCD chips with a baseline of $7.6 \mathrm{~mm}$. The module provides 3D vision feedback with controllable zoom, pan and tilt for increased visual field, Fig. 2. The aim of this 3D vision feedback is to provide depth perception to the surgeon and to provide automatic instrument tracking (e.g.[17]). Other planned applications of this module include online estimation of flexible robot actuation compensation parameters (e.g.[18]).

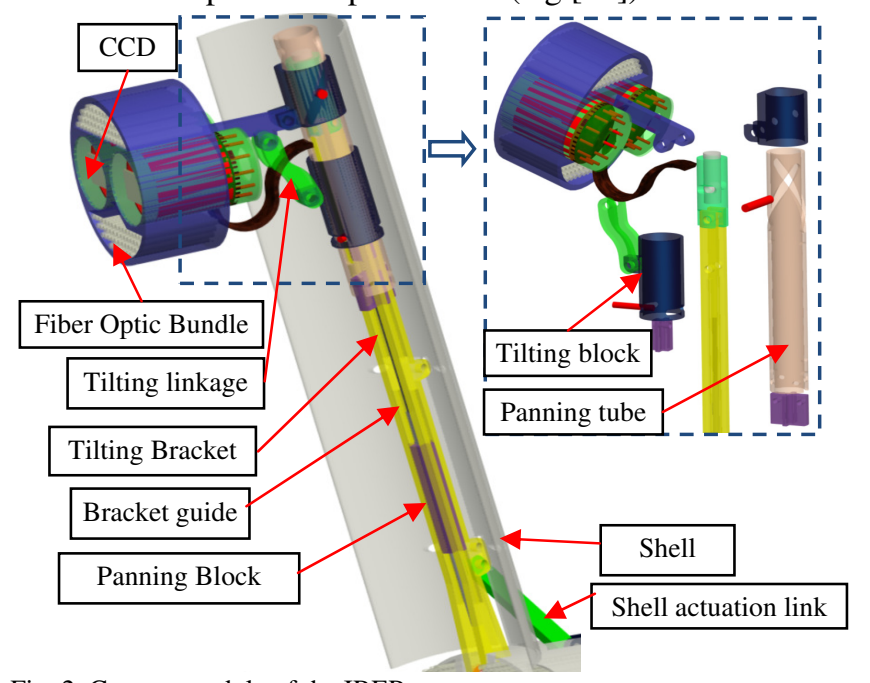

Fig. 2. Camera module of the IREP

The vision module is controlled by $3 \mathrm{DoF}$ arms that provide pan, tilt and zoom movement. The zoom functionality is achieved by opening and closing the controllable shell. Pan is achieved by linear actuation of the panning block to drive the relative movement between the panning tube and the bracket guide. The panning tube can generate panning movement via its helical grooves. The tilting movement is also actuated using push-pull actuation of the tilting block, which drives the 
tilting linkage to generate camera tilting movement.

The actuation unit of the IREP includes several sub-modules: one robot base, two motor housings, and two identical snake actuation units (Fig. 3). The base module carries fixed components (vision module and two five-bar mechanisms). The base module also carries all motors for the IREP and it provides gross axial motion along the axis of the IREP lumen. The motor housing carries all the motors and it connects to each dexterous arm via a quick-connect interface equipped with six Oldham couplings. This design removes all the motors from the snake-arm actuation units in order to reduce weight and to simplify interchangeability of robotic arms. The snake actuation unit includes four twin-lead screws for actuating the two-segment continuum robot and two lead screws to actuate the distal wrist and gripper. The distal wrist is wire-actuated and the gripper is actuated by a NiTi wire.
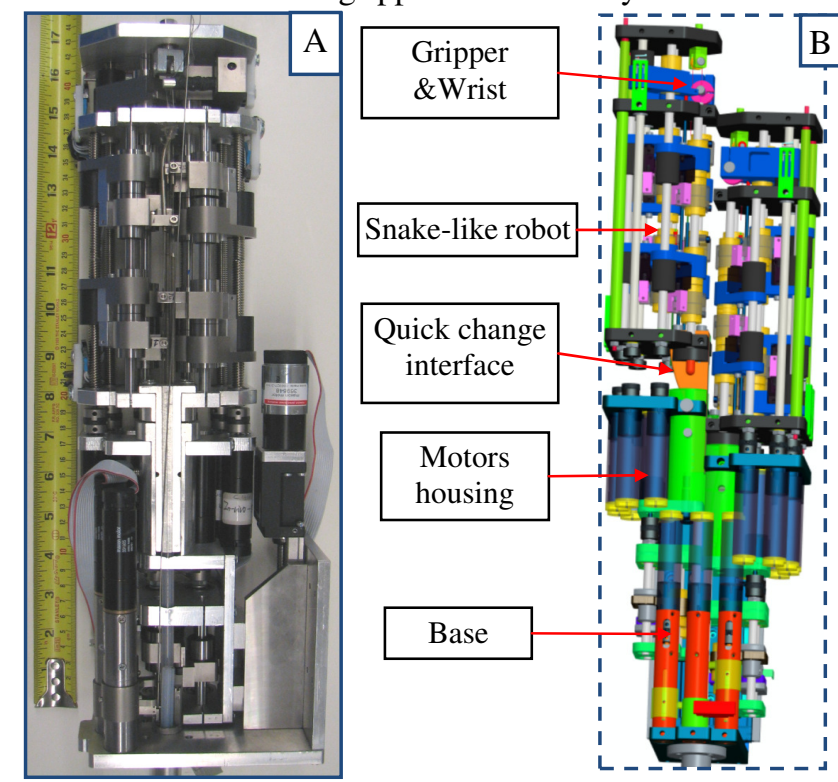

Fig. 3. ProE model and prototype of Actuation unit

The IREP weighs less than $4.5 \mathrm{~kg}$ and its actuation unit fits within a 70x140x 220mm volume. The actuation unit of each dexterous arm weighs $2.25 \mathrm{~kg}$.

\section{KINEMATIC AND STATIC MODELING OF THE IREP}

Fig. 4 and the list below define necessary nomenclature for the formulation of the kinematics and statics of the IREP. Since both arms of the IREP are identical, only the kinematics of one dexterous arm is presented.

- $\{\mathrm{A}\}$ - A right handed frame with $\left\{\hat{\mathbf{x}}_{a}, \hat{\mathbf{y}}_{a}, \hat{\mathbf{z}}_{a}\right\}$ as its axes and point $\mathbf{a}$ as its origin .

- ${ }^{\mathrm{A}} \mathbf{p}_{\mathrm{a} / \mathrm{b}}$ position vector from point $\mathbf{b}$ to $\mathbf{a}$ expressed in $\{\mathrm{A}\}$.

- ${ }^{\mathrm{A}} \mathbf{R}_{\mathrm{B}}$ - orientation of frame $\{\mathrm{B}\}$ relative to $\{\mathrm{A}\}$

- ${ }^{\mathrm{C}} \mathbf{v}_{\mathrm{A} / \mathrm{B}},{ }^{\mathrm{C}} \boldsymbol{\omega}_{\mathrm{A} / \mathrm{B}}$ - linear and angular velocity of frame $\{\mathrm{A}\}$ with respect to frame $\{B\}$ expressed in frame $\{C\}$.

- ${ }^{\mathrm{C}} \mathbf{t}_{\mathrm{A} / \mathrm{B}}=\left[{ }^{\mathrm{C}} \mathbf{v}_{\mathrm{A} / \mathrm{B}},{ }^{\mathrm{C}} \boldsymbol{\omega}_{\mathrm{A} / \mathrm{B}}\right]$ - twist of frame $\{\mathrm{A}\}$ with respect to $\{B\}$ expressed in $\{C\}$. Unless otherwise stated, all twists are defined in base frame $\left\{B_{0}\right\}$.
- $\left[\mathbf{p}^{\wedge}\right]$-the skew-symmetric cross product matrix of vector $\mathbf{p}$.

- $\theta_{i}$ - the angle describing the bending of the $\mathrm{i}^{\text {th }}$ segment from its straight configuration $\theta_{i}=\pi / 2$, Fig. 4-(c).

- $\delta_{i}$ - the angle describing the plane in which the $\mathrm{i}^{\text {th }}$ segment $(i=1,2)$ bends. This angle is defined from the bending plane to the first backbone. The direction of $\delta_{i}$ is defined by the right-hand rule about $\hat{\mathbf{z}}_{b i}$.

- $\boldsymbol{\eta}=\left(q_{1}, q_{2}, \theta_{1}, \delta_{1}, \theta_{2}, \delta_{2}, q_{7}\right)^{\mathrm{T}}$ - configuration variables, $q_{1}, q_{2}$ are the joint values of the parallelogram mechanism. $q_{7}$ is the wrist rotation angle measured according to the right-hand rule about $\hat{\mathbf{z}}_{t}$. All joint values are defined with respect to a home configuration in which the dexterous arm is straight and q7=0 as defined in Fig. 4-(b).

- $\mathrm{L}_{i}$ - length of the primary backbone of the $\mathrm{i}^{\text {th }}$ segment.

- $\mathrm{d}_{i}-\mathrm{i}=1 . .4$, the lengths of the links for the parallelogram linkage: $\mathrm{d} 1=|\mathrm{p} 5-\mathrm{p} 2|=|\mathrm{p} 6-\mathrm{p} 3|=35 \mathrm{~mm}, \mathrm{~d} 2=|\mathrm{p} 5-\mathrm{p} 6|=|\mathrm{p} 2-\mathrm{p} 3|=2$. $3 \mathrm{~mm}, \mathrm{~d} 3=|\mathrm{p} 2-\mathrm{p} 4|=\mathrm{d} 1 / 2, \mathrm{~d} 4=|\mathrm{p} 4-\mathrm{p} 1|=20 \mathrm{~mm}$.

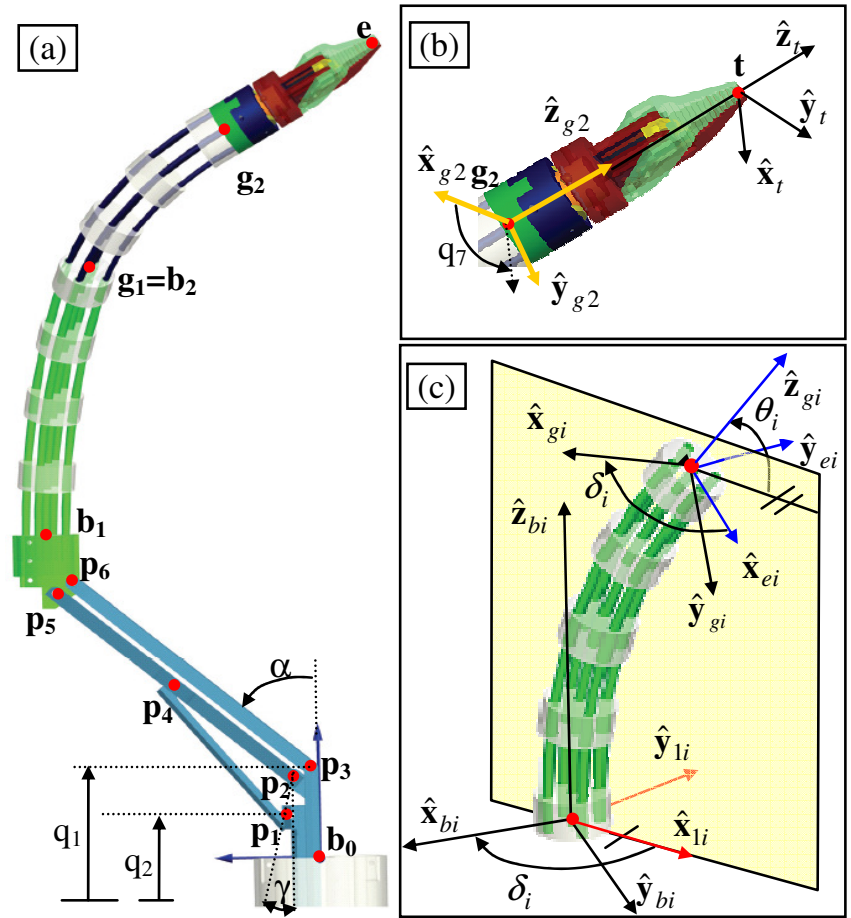

Fig. 4. Nomenclature of a single dexterous arm of the IREP: (a) definition of points, (b) definition of the tip frame and the wrist rotation angle $q_{7}$, (c) definition of local frames for the $\mathrm{i}^{\text {th }}$ segment of the continuum robot TABLE 2 SPAS ROBOT GEOMETRIC DIMENSION

\begin{tabular}{|c|c|c|c|c|}
\hline \multicolumn{5}{|c|}{ Snake geometric dimension (mm) } \\
\hline $\begin{array}{c}1^{\text {st }} \text { stage } \\
\text { length }\end{array}$ & $\begin{array}{c}2^{\text {nd }} \text { stage } \\
\text { length }\end{array}$ & $\begin{array}{c}\text { Gripper } \\
\text { length }\end{array}$ & $\begin{array}{c}\text { Disk } \\
\text { Dia }\end{array}$ & $\begin{array}{c}\text { Disk } \\
\text { Height }\end{array}$ \\
\hline 35 & 25 & 15 & 6.4 & 3.2 \\
\hline \multicolumn{5}{|c|}{ SPAS robot Joint ranges } \\
\hline$q_{1} \mathrm{~mm}$ & $q_{2} \mathrm{~mm}$ & $\theta_{1,2} \operatorname{deg}$ & $\delta_{1,2} \mathrm{deg}$ & $q_{7} \mathrm{deg}$ \\
\hline $0 \sim 80$ & $0 \sim 30$ & $\pm 90^{\circ}$ & $\pm 180^{\circ}$ & $\pm 90^{\circ}$ \\
\hline
\end{tabular}

\section{A. Position analysis of the IREP}

A base frame $\left\{B_{0}\right\}$ is defined at the tip of the central stem, Fig. 4-(a). The direct kinematics of each dexterous arm is 
described using its augmented configuration variables $\boldsymbol{\eta}$. The position of the gripper with respect to $\left\{\mathrm{B}_{0}\right\}$ is given by:

$$
{ }^{\mathrm{B} 0} \mathbf{p}_{\mathrm{e} / \mathrm{b}_{0}}={ }^{\mathrm{B} 0} \mathbf{p}_{\mathrm{b}_{1} / \mathrm{b}_{0}}+{ }^{\mathrm{B} 0} \mathbf{p}_{\mathrm{g}_{1} / \mathrm{b}_{1}}+{ }^{\mathrm{B} 0} \mathbf{R}_{\mathrm{G} 1}{ }^{\mathrm{G} 1} \mathbf{p}_{\mathrm{g}_{2} / \mathrm{b}_{2}}+{ }^{\mathrm{B}_{0}} \mathbf{R}_{\mathrm{G}_{2}}{ }^{{ }_{\mathrm{G}}} \mathbf{p}_{\mathrm{e} / \mathrm{g}_{2}}
$$

The vectors ${ }^{\mathrm{B}_{0}} \mathbf{p}_{\mathrm{b}_{1} / \mathrm{b}_{0}},{ }^{\mathrm{B}_{0}} \mathbf{p}_{\mathrm{g}_{1} / \mathrm{b}_{1}},{ }^{\mathrm{G}_{1}} \mathbf{p}_{\mathrm{g}_{2} / \mathrm{b}_{2}}$ are defined by Eqs. (1), (3) and ${ }^{\mathrm{G} 2} \mathbf{p}_{\mathrm{t} / \mathrm{g}_{2}}=\left\|\mathbf{e}-\mathbf{g}_{2}\right\| \mathbf{e}_{3}$.

Using the order $O=[b 0<b 1<g 1<g 2<e]$, the rotation matrices in Eq. (1) are given by:

$$
{ }^{k} \mathbf{R}_{j}={ }^{k} \mathbf{R}_{k+1}{ }^{k+1} \mathbf{R}_{k+1} \cdots{ }^{j-1} \mathbf{R}_{j} \text { where } i, j \in O
$$

Explicitly, ${ }^{\mathrm{G} 2} \mathbf{R}_{\mathrm{E}}=\mathbf{e}^{q_{7}\left[\mathrm{e}^{\wedge}{ }^{\wedge}\right]}$, and the segment rotation matrices ${ }^{\mathrm{G}_{1}} \mathbf{R}_{\mathrm{B}_{1}}$ and ${ }^{\mathrm{G}_{2}} \mathbf{R}_{\mathrm{B}_{2}}$ are defined by Eq. (8).

The direct kinematics of the parallelogram is given by

$$
{ }^{\mathrm{B} 0} \mathbf{p}_{\mathrm{b}_{1} / \mathrm{b}_{0}}=\left[\begin{array}{c}
\mathrm{d}_{1} \sin (\alpha)+\mathrm{u}_{1} \\
0 \\
q_{1}+\mathrm{d}_{1} \cos (\alpha)+\mathrm{u}_{2}
\end{array}\right], \quad{ }^{B 0} \mathbf{R}_{\mathrm{B} 1}=\mathbf{I}_{3 x 3}
$$

Where $\left(\mathbf{b}_{1}-\mathbf{p}_{6}\right)=\left[\mathrm{u}_{1}, 0, \mathrm{u} 2\right]^{\mathrm{T}}$ and $\alpha$ is the parallelogram tilt angle as in Fig. 4-(a). This angle is given by the law of cosines for $\mathbf{p}_{1}, \mathbf{p}_{2}, \mathbf{p}_{4}$ while considering the offset angle $\gamma$.

$$
\begin{gathered}
\gamma=\tan ^{-1}\left(\mathrm{u}_{3} /\left(q_{1}-q_{2}\right)\right) \\
\alpha=\cos ^{-1} \underbrace{\left(\frac{\mathrm{d}_{4}{ }^{2}-\mathrm{d}_{3}{ }^{2}-\left(\left(q_{1}-q_{2}\right)^{2}+\mathrm{u}_{3}{ }^{2}\right)}{2 \mathrm{~d}_{3} \sqrt{\left(q_{1}-q_{2}\right)^{2}+\mathrm{u}_{3}{ }^{2}}}\right)}_{\beta}-\gamma
\end{gathered}
$$

where

$$
u_{3}=\left(\mathbf{p}_{1}-\mathbf{p}_{2}\right)^{T} \hat{x}_{b_{0}}
$$

The direct and inverse kinematics of each segment of the continuum robot was described in[19]. The position and orientation of the end disk of the $i^{\text {'th }}$ segment with respect to its base are respectively given by ${ }^{\mathrm{B}_{i}} \mathbf{p}_{\mathrm{e}_{i} / \mathrm{b}_{i}}$ and ${ }^{\mathrm{B}_{i}} \mathbf{R}_{\mathrm{G}_{i}}$ :

$$
\begin{gathered}
{ }^{\mathrm{B}_{i}} \mathbf{p}_{\mathrm{e}_{i} / b_{i}}=\mathrm{L}_{i} /\left(\frac{\pi}{2}-\theta_{i}\right) e^{-\delta_{i}\left[\mathrm{e}^{\wedge}{ }^{\wedge}\right.}\left[1-\sin \left(\theta_{i}\right), 0, \cos \left(\theta_{i}\right)\right]^{\mathrm{T}} \\
{ }^{\mathrm{B}_{i}} \mathbf{R}_{\mathrm{G}_{i}}=e^{-\delta_{i}\left[\mathrm{e}_{3}{ }^{\wedge}\right]} e^{\left(\frac{\pi}{2}-\theta_{i}\right)\left[\mathrm{e}_{2}{ }^{\wedge}\right]} e^{\delta_{i}\left[\mathrm{e}_{3}{ }^{\wedge}\right]}
\end{gathered}
$$

where $\mathbf{e}_{i}(\mathrm{i}=1,2,3)$ are basis unit vectors for $\mathbb{R}^{3 \times 1}$.

\section{B. Instantaneous kinematics of the IREP}

Let matrices $\mathbf{J}_{p}, \mathbf{J}_{x \psi_{i}}(\mathrm{i}=1,2)$, and $\mathbf{J}_{w}$ respectively denote the Jacobians of the parallelogram, the i'th segment of the snake arm, and the wrist. The parallelogram Jacobian, $\mathbf{J}_{\mathrm{p}}$, is given by differentiating Eq. (5) with respect to time:

$$
\mathrm{B}_{0} \mathbf{t}_{\mathrm{B}_{1} / \mathrm{B}_{0}}=\underbrace{\left[\begin{array}{cc}
\frac{\partial \alpha}{\partial q_{1}}\left(\mathrm{~d}_{1} c_{\alpha} \hat{\mathbf{e}}_{1}-s_{\alpha} \hat{\mathbf{e}}_{3}\right)+\hat{\mathbf{e}}_{3} & \frac{\partial \alpha}{\partial q_{2}}\left(\mathrm{~d}_{1} c_{\alpha} \hat{\mathbf{e}}_{1}-\mathrm{d}_{1} s_{\alpha} \hat{\mathbf{e}}_{3}\right) \\
\mathbf{0}_{3 \times 1} & \mathbf{0}_{3 \times 1}
\end{array}\right]}_{\mathbf{J}_{p}}\left[\begin{array}{c}
\dot{q}_{1} \\
\dot{q}_{2}
\end{array}\right] \text { (9) }
$$

where the derivative of $\alpha$ is given by:

$$
\frac{\partial \alpha}{\partial \mathrm{q}_{1}}=-\frac{1}{\sqrt{1-\beta^{2}}} \frac{d \beta}{d q_{1}}+\frac{\mathrm{u}_{3}}{\left(q_{1}-q_{2}\right)^{2}+\mathrm{u}_{3}^{2}}
$$

$$
\frac{\partial \alpha}{\partial \mathrm{q}_{2}}=-\frac{1}{\sqrt{1-\beta^{2}}} \frac{d \beta}{d q_{2}}-\frac{\mathrm{u}_{3}}{\left(q_{1}-q_{2}\right)^{2}+\mathrm{u}_{3}^{2}}
$$

The Jacobian of each segment was given by [19] using a subsequent mapping from task space to configuration space and from configuration space to joint space:

${ }^{\mathrm{B}_{0}} \mathbf{t}_{G_{i} / B_{i}}=\mathbf{E} \mathbf{J}_{x \psi_{i}} \dot{\boldsymbol{\psi}}_{i}$ where $i=1,2 \mathbf{E}=\left[\begin{array}{cc}{ }^{\mathrm{B}_{0}} \mathbf{R}_{\mathrm{B}_{i}} & \mathbf{0}_{3 \times 3} \\ \mathbf{0}_{3 \times 3} & { }^{\mathrm{B}_{0}} \mathbf{R}_{\mathrm{B}_{i}}\end{array}\right]$

Where $\mathbf{J}_{x \psi_{i}}(\mathrm{i}=1,2)$ is the Jacobian relating the configuration speeds $\dot{\psi}_{i}=\left[\dot{\theta}_{i}, \dot{\delta}_{i}\right]^{\mathrm{T}}$ to the relative twist of the end disk with respect to the base disk of the $i^{\text {th }}$ segment.

The Jacobian of the wrist is given by

$$
{ }^{\mathrm{B}_{0}} \mathbf{t}_{E / \mathrm{G}_{2}}=\left[\begin{array}{c}
\mathbf{0}_{3 \times 1} \\
{ }^{\mathrm{B}} 0 \\
\mathbf{R}_{E} \hat{\mathbf{e}}_{3}
\end{array}\right] \cdot \dot{q}_{7}=\mathbf{J}_{w} \cdot \dot{q}_{7}
$$

Equations 9, 12, 13 provide the gripper twist:

$$
{ }^{\mathrm{B}_{0}} \mathbf{t}_{E / \mathrm{B}_{0}}={ }^{\mathrm{B}_{0}} \mathbf{t}_{\mathrm{B}_{1} / \mathrm{B}_{0}}+\mathbf{S}_{1}{ }^{\mathrm{B}_{0}} \mathbf{t}_{\mathrm{G}_{1} / \mathrm{B}_{1}}+\mathbf{S}_{2}{ }^{\mathrm{B}_{0}} \mathbf{t}_{\mathrm{G}_{2} / \mathrm{B}_{2}}+{ }^{\mathrm{B}_{0}} \mathbf{t}_{\mathrm{E}_{\mathrm{G}} / \mathrm{G}_{2}}
$$

where twist transformation matrices $\mathrm{S} 1$ and $\mathrm{S} 2$ are given by:

$$
\mathbf{S}_{1}=\left[\begin{array}{cc}
\mathbf{I}_{3 \times 3} & \left(\left[{ }^{\mathrm{B}_{0}} \mathbf{p}_{g_{1} / e^{\wedge}}\right]\right) \\
\mathbf{0}_{3 \times 3} & \mathbf{I}_{3 \times 3}
\end{array}\right] \mathbf{S}_{2}=\left[\begin{array}{cc}
\mathbf{I}_{3 \times 3} & {\left[{ }^{\mathrm{B}} \mathbf{p}_{g_{2} / e^{\wedge}}\right]} \\
\mathbf{0}_{3 \times 3} & \mathbf{I}_{3 \times 3}
\end{array}\right]
$$

Hence the Jacobian of a single arm of the IREP is:

$$
\mathbf{J}=\left\lfloor\begin{array}{llll}
\mathbf{J}_{1}, & \mathbf{S}_{1} \mathbf{J}_{x \psi_{1}}, & \mathbf{S}_{2} \mathbf{J}_{x \psi_{2}}, & \mathbf{J}_{w}
\end{array}\right\rfloor
$$

\section{Statics modeling and Actuation force Estimation}

The static analysis of the IREP is based on the virtual work principle. Here we make following assumptions: 1) The continuum robots assume a shape determined by their minimum potential energy (it is assumed that the dynamic forces may be neglected at low speeds typical to surgical applications). 2) The elastic energy $\mathrm{E}$ is mainly due to the bending of the backbones. Significant twisting of the backbones does not occur in normal operation of the continuum robot. 3) Although out-of plane bending may occur due to large forces at the tip of the snake, our assumption is that the deflections caused by these forces are small enough (otherwise, the continuum robot is too flexible and ineffective for surgical tasks). 4) We also ignore all the internal energy dissapation caused by friction.

The potential energy $P$ includes the elastic energy $E$ of the backbones and the gravitational potential energy $\mathrm{G}$ due to the weight of the disks and gripper. Hence, we assume in-plane bending neglect twisting of the backbones.

$$
\begin{aligned}
& E=\sum_{i=1}^{2} \frac{\left(\theta_{i}-\pi / 2\right)^{2}}{2}\left(\frac{E_{p_{i}} I_{p_{i}}}{L}+\sum_{j=1}^{4} \frac{E_{s_{i}} I_{s_{i}}}{\left(L_{j}+r c_{\delta_{j}}\right)\left(\theta_{i}-\pi / 2\right)}\right) \\
& G=\sum_{i=1}^{2} \frac{L_{i}\left(-g \mathbf{e}_{3}^{\mathrm{T}}\right)}{\left(\theta_{i}-\pi / 2\right)}\left(\mathbf{R}_{0} \mathbf{R}_{i} e^{-\delta_{i}\left[\mathbf{e}_{3}{ }^{\wedge}\right]} \sum_{j=1}^{n} m_{j}\left[\left(1-c_{\beta_{j}}\right), 0, s_{\beta}\right]_{j}^{\mathrm{T}}\right)
\end{aligned}
$$

$\mathbf{R}_{0}$ is the orientation of the central stem in Fig. 1 with respect to the world frame, and $\beta_{j}=(j / n)\left(\pi / 2-\theta_{i}\right)$ is an angular coordinate used to calculate the position of disk $j$ along the backbone. Since the weight of each disk is $0.66 \mathrm{~g}$ and the 
gripper weight $0.72 \mathrm{~g}$, the gravitational potential energy is negligible compared to the elastic energy [20].

To estimate the required actuation forces, a sweep of the workspace of the IREP arm was conducted while subjecting the gripper to forces in a plane perpendicular to its longitudinal axis. The norm of these forces was assumed to be $2 \mathrm{~N}$ in accordance with our design specifications in Table I. The required actuation forces were estimated using a worst-case scenario in which the first segment is bent in the range $\psi \in([0, \pi / 2],[-\pi, \pi])$ while maintaining the second segment fully extended $\left(\theta_{2}=\pi / 2\right)$.

The actuation forces $\tau$ required to balance an external wrench $\mathbf{W}_{\mathrm{e}}=\left[\begin{array}{ll}\mathbf{f}_{e}^{T}, & \mathbf{m}_{e}^{T}\end{array}\right]^{T}$ are given by [21]:

$$
\boldsymbol{\tau}=\mathbf{J}_{\mathrm{q} \psi}^{+}\left(\nabla \mathbf{E}+\nabla \mathbf{G}-\mathbf{J}_{\mathrm{x} \psi}^{\mathrm{T}} \mathbf{W}_{\mathrm{e}}\right)
$$

Fig. 5 shows a maximal actuation force of $56.2 \mathrm{~N}$. Hence, the required actuation unit force is $60 \mathrm{~N}$ as shown in Table 1.

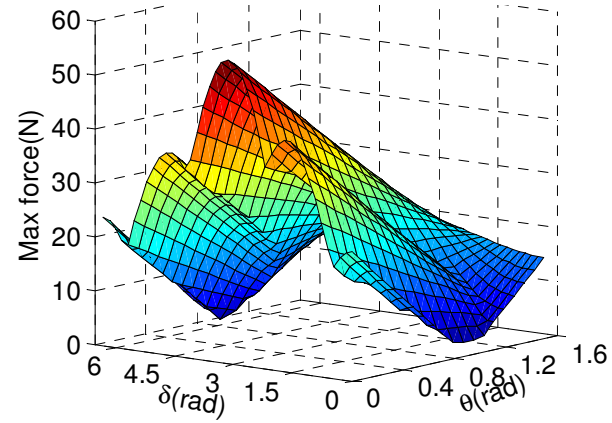

Fig. 5. Estimation of the required actuation force

\section{IREP SUTURING AND PERFORMANCE EVALUATION}

\section{A. Evaluation of Dual-arm Suturing Capability}

The dexterity of the IREP was verified for passing circular suturing needles at multiple locations along a sinusoidal path in the XY cross section of the desired workspace, Fig. 6. The path had amplitude of $4 \mathrm{~mm}$ and a wave length of $40 \mathrm{~mm}$. At each point along the path, the IREP inserts a $3 / 8$ circular needle (diameter $16 \mathrm{~mm}$ ) through $100^{\circ}$ rotation. To minimize tissue trauma, the IREP keeps the needle plane perpendicular to the curve local tangent.

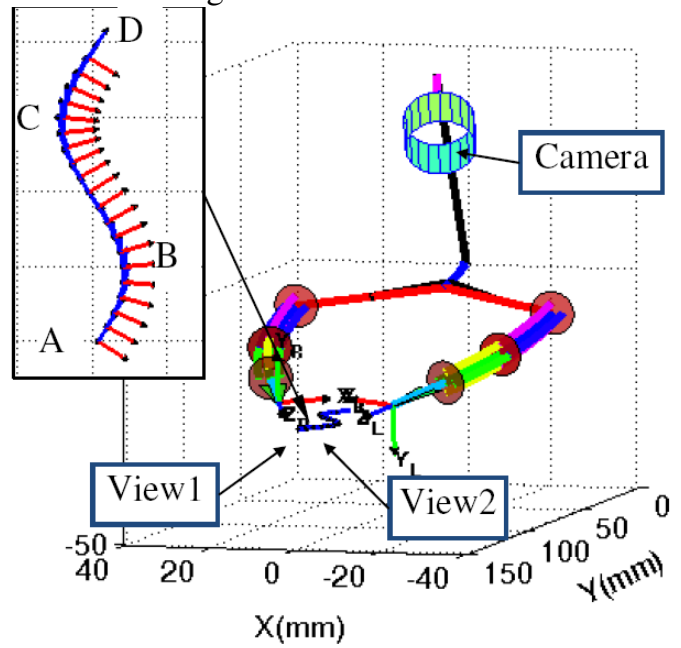

Fig. 6. SPAS robot dual arm pass suture.
Fig. 7 and the multimedia extension show the IREP passing a circular needle at $0^{\circ}, 45^{\circ}$, and $90^{\circ}$ feeding angle along this sinusoidal path of Fig. 6. The handedness during suturing of this curve was selected for maximum dexterity. For example, the curve tangent along $\mathrm{BC}$ segment (Fig. 6) is easier to execute using the right hand. Using similar arguments, the left hand is more suitable for passing suturing needles at points along the $\mathrm{AB}$ and $\mathrm{CD}$ curve segments.

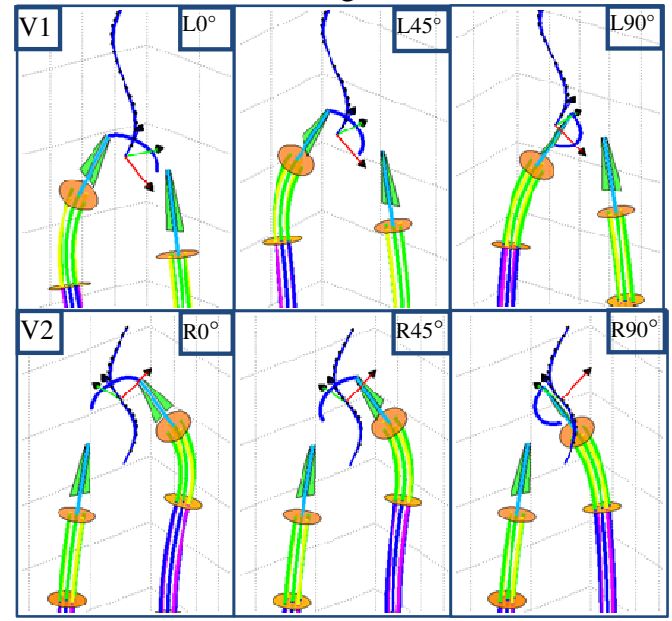

Fig. 7. Suturing simulation: (Top) left-hand suturing as seen from view 1 in Fig. 6, (Bottom) right-hand suturing as seen from view 2 in Fig. 6. In order to keep a fair comparison between design alternatives, the control of the redundant IREP did not involve any optimization criteria. The minimal-norm pseudo inverse solution to minimize joint movements was used:

$$
\dot{\boldsymbol{\eta}}=\mathbf{J}^{+} \mathbf{t}_{\mathrm{E} / \mathbf{B}_{\mathbf{0}}}
$$

\section{B. Design Justification of distal wrist}

Though the IREP has a distal wrist, it is possible to pass circular needles by using the continuum robot to transmit rotation from its base to its gripper [16]. We carried out a simulation comparing the dexterity of these two alternative designs while performing the task of suturing along the path of Fig. 6. Fig. 8 shows the Kinematic Conditioning Index (KCI) defined as the ratio of the minimal and maximal singular values of the Jacobian. The KCI is compared in this figure for both design alternatives. The figure shows that the addition of the distal wrist pays off mainly in increased translational dexterity since the backbones have to otherwise move faster to satisfy the synchronous movement required for transmitting rotation about the snake backbone.

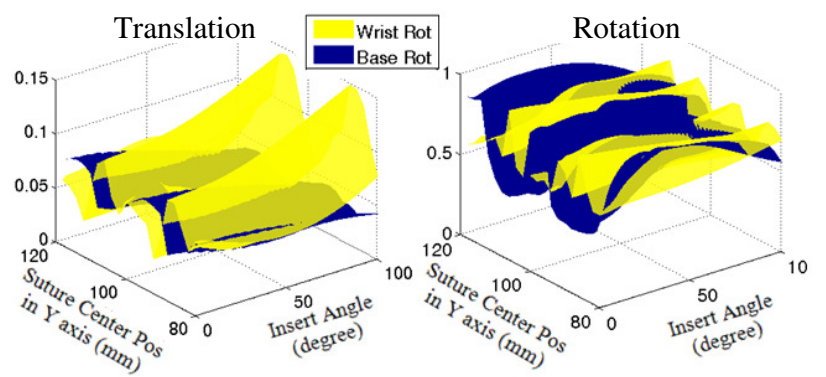

Fig. 8. Translational and rotational dexterity comparison of a design with a distal wrist (Wrist Rot) with a design that uses backbone rotation (Base Rot). 


\section{Design justification of parallelogram mechanisms}

Another design alternative for the IREP includes kinematic arrangements similar to [4] and [6], which do not have specific mechanism to deploy the two arms and have both dexterous arms with a minimal fixed offset. It is possible to compare the effects of adding the offset between the bases of each snake arm by assuming both parallelograms locked at a specific offset distance. Fig. 9 shows the dexterity advantages of using a larger offset between the IREP arms while performing the task of suturing in Fig. 6.

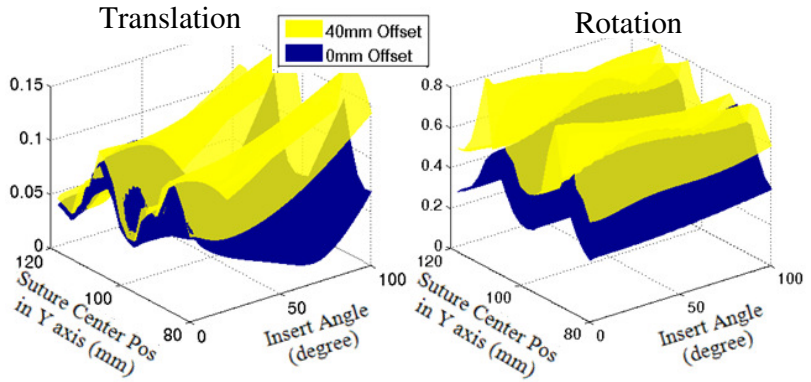

Fig. 9. Translational and Rotational dexterity comparison of the IREP with $0 \mathrm{~mm}$ and $40 \mathrm{~mm}$ offset between the bases of each IREP snake arm.

Table 3 shows the average KCI of the IREP and the alterative design with zero offset. These results justify the use of a distal wrist and parallelogram linkages for dexterity benefits.

TABLE 3 COMPARISON KCI WHILE PERFORMING SUTURE TASK

\begin{tabular}{|c|c|c|c|c|}
\hline KCI & IREP & Base rot & 0mm Offset & $40 \mathrm{mmOffset}$ \\
\hline Translational & 0.0975 & 0.0496 & 0.0530 & 0.0735 \\
\hline Rotational & 0.6793 & 0.6900 & 0.4472 & 0.6444 \\
\hline
\end{tabular}

\section{CONCLUSION}

This paper presented an Insertable Robotic Effectors Platform (IREP) for Single Port Access Surgery (SPAS). The unique design of the IREP allows it to fit through a $\varnothing 15 \mathrm{~mm}$ port while providing $3 \mathrm{D}$ vision feedback to guide insertion and deployment of two dexterous arms with a controllable stereo vision module. Modeling of the kinematics and statics of the IREP was presented and a benchmark task of dual-arm suturing along a predetermined sinuous curve was simulated to compare several design alternatives. Results of these simulations showed that the IREP has improved dexterity for suturing when rotation about the longitudinal axis of its gripper is achieved via an independent distal rotation joint instead of transmittal of rotation about the backbone of the continuum robot. The effect of changing the base distance between the two snake arms was evaluated and it was shown that designs with the same continuum robots as dexterous arms have diminished dexterity when both arms overlap at their bases. The simulation showed also that certain paths are better performed by the left hand or the right hand of the IREP. Future work will include integration of vision feedback for the telemanipulation control of the IREP and experimental evaluation on phantom models.

\section{REFERENCES}

[1] R. Taylor and D. Stoianovici, "Medical Robotics in Computer-Integrated Surgery," IEEE Transactions on Robotics and Automation, vol. 19, pp. 765-781, 2003.

[2] G. Guthart and K. Salisbury, "The IntuitiveTM Telesurgery System: Overview and Application," in IEEE International Conference on Robotics and Automation San Francisco, 2000, pp. 618-621.

[3] M. Bessler, P. D. Stevens, L. Milone, M. Parikh, and D. Fowler, "Transvaginal laparoscopically assisted endoscopic cholecystectomy: a hybrid approach to natural orifice surgery," Gastrointestinal Endoscopy, vol. 66, pp. 1243-1245, 2007.

[4] D. J. Abbott, C. Becke, R. I. Rothstein, and W. J. Peine, "Design of an endoluminal NOTES robotic system," in IEEE International Conference - IROS, San Diego, 2007, pp. 410-416.

[5] A. C. Lehman, N. A. Wood, J. Dumpert, D. Oleynikov, and S. M. Farritor, "Robotic natural orifice translumenal endoscopic surgery," in IEEE International Conference on Robotics and Automation Pasadena, 2008, pp. 2969-2974.

[6] S. J. Phee, S. C. Low, Z. L. Sun, K. Y. Ho, W. M. Huang, and Z. M. Thant, "Robotic system for no-scar gastrointestinal surgery," The International Journal of Medical Robotics and Computer Assisted Surgery, vol. 4, pp. 15-22, 2008.

[7] M. F. Saad, "Fisherman's Technique, Introducing a Novel Method for Using the Umbilical Port for Removal of Appendix During Laparoscopic Appendectomy," Surg Laparosc Endosc Percutan, vol. 17, pp. 422-424, 2007.

[8] A. A. Gumbs, L. Milone, and P. Sinha, "Totally Transumbillical Laparoscopic Cholecystectomy," Journal of Gastrointestinal Surgery, vol. 3, pp. 533-534, 2008 .

[9] J. R. Romanelli, "Single-port laparascopic surgery: an overview," Surg Endosc, vol. DOI 10.1007/s00464-009-0463-x, 2009.

[10] J. Romanelli and D. Earle, "Single-port laparoscopic surgery: an overview," Surgical Endoscopy, vol. 23, pp. 1419-1427, 2009.

[11] A. Gumbs, L. Milone, P. Sinha, and M. Bessler, "Totally Transumbilical Laparoscopic Cholecystectomy," Journal of Gastrointestinal Surgery, 2008.

[12] K.Harada, E. Susilo, A.Menciassi, and P. Dario, "Wireless reconfigurable modules for robotic endoluminal surgery," in IEEE International Conference on Robotics and Automation, Japan, 2009, pp. $2699-2704$.

[13] K. Xu, R. E. Goldman, J. Ding, P. K. Allen, D. L. Fowler, and N. Simaan, "System Design of an Insertable Robotic Effector Platform for Single Port Access (SPA) Surgery," in IEEE International Conference IROS St. Louise 2009, pp. 5546-5552.

[14] A. Dubrowski, R. Sidhu, J. Park, and H. Carnahan, "Quantification of motion characteristics and forces applied to tissues during suturing," The American Journal of Surgery, vol. 190, pp. 131-136, 2004.

[15] J. Peirs, J. Clijnen, D. Reynaerts, H. V. Brussel, P. Herijgers, B. Corteville, and S. Boone, "A micro optical force sensor for force feedback during minimally invasive robotic surgery," Sensors and Actuators A: Physical, vol. 115, pp. 447-455, 2004.

[16] N. Simaan, X. Kai, W. Wei, A. Kapoor, P. Kazanzides, R. Taylor, and P. Flint, "Design and Integration of a Telerobotic System for Minimally Invasive Surgery of the Throat," The International Journal of Robotics Research, vol. 28, pp. 1134-1153, 2009.

[17] H. Tie, P. K. Allen, T. Nadkarni, N. J. Hogle, and D. L. Fowler, "Insertable stereoscopic 3D surgical imaging device with pan and tilt," in 2nd IEEE RAS \& EMBS International Conference on Biomedical Robotics and Biomechatronics(BioRob) Arizona, 2008, pp. 311-316.

[18] K. Xu and N. Simaan, "Actuation Compensation for Flexible Surgical Snake-like Robots with Redundant Remote Actuation " in IEEE International Conference on Robotics and Automation Orlando, 2006, pp. 4148-4154.

[19] N. Simaan, R. Taylor, and P. Flint, "A dexterous system for laryngeal surgery," in IEEE International Conference on Robotics and Automation New Orleans, 2004, pp. 351-357.

[20] X. Kai and N. Simaan, "An Investigation of the Intrinsic Force Sensing Capabilities of Continuum Robots," Robotics, IEEE Transactions on, vol. 24, pp. 576-587, 2008

[21] N. Simaan, "Snake-Like Units Using Flexible Backbones and Actuation Redundancy for Enhanced Miniaturization," in IEEE International Conference on Robotics and Automation, Barcelona, Spain, 2005, pp. 3020-3028. 